\title{
BARRIERS IN THE MATHEMATICAL MODELLING OF DECISION-MAKING
}

\author{
Popescu Oana ${ }^{1}$
}

\begin{abstract}
Decision-making is becoming increasingly complex as the number of stakeholders rises, the number of decision-makers rises as well, the implications to consider are various and farreaching, risks and uncertainties abound and the regulatory framework is growing increasingly complex. Decision-making is caught between the need to thoroughly document and substantiate a choice in front of oneself and others, on one hand, and the potential traps that increase in number and impact as the complexity of the decision-making process increases, on the other hand. The present paper sought to present critically the main types of downfalls that a decision-making model may face at the level of an individual decision-maker. Our approach was to put into relation some of the most notable contributions from various perspectives in order to obtain an integrated overview of the challenges faced by decision sciences in formulating a both thorough and life-like decision-making model. We identified with this occasion three main potential weaknesses in formal decision modeling: unquantifiable factors, such as feelings or morals, excessive formalism and simplification of models that make them vulnerable to paradoxes, and finally cognitive biases that deviate models from an objective path. Though scientific literature on each of these topics abounds, it is time to integrate it and set the basis for comprehensive and multi-perspective modeling of decisions.
\end{abstract}

Key words: decision, decision-making, decision theory, game theory, economic modeling

JEL Codes: C18, C51, C70, D70, D80, D90

\section{Introduction}

Mathematical models, matrixes and equations are regarded by some as the exclusive instruments of exact sciences such as mathematics, physics with all its ramifications, chemistry and the like. The degree of real-life applicable structuring and abstractisation of problems depends on each individual's predisposition and even academic formation in exact or humanistic sciences. Whether a general best practice can be prescribed is still a matter of debate among scholars. No sooner had the first attempts to define the decision-making process been formulated that critics were addressed from all perspectives. While initially basing his research on the paradigm of ,economic man”, or „homo economicus”, as a perfectly rational creature whose only goal is to maximize his own utility through extensive and coherent cognitive processes, Simon is among the most notable detractors of this construct (Simon, 1957). Instead, he uses uncertainty and bounded rationality to define ,organizational man”. Unlike economic man, organizational man does not display a perfect rationality, but rather a bounded one. Unlike economic man, organizational man does not seek to optimize, but rather to find a satisfactory outcome, to ,satisfice”. However, the scientific approach to problem-solving is maintained through clearly specified objectives, well-defined goals, and a continuous bearing in mind of the problem overview while participants to the decision-making process seek satisfactory or sufficient solutions in addressing the problem. More extreme critics completely by-pass problem-solving approaches due to overwhelming interference caused by

\footnotetext{
1 The Bucharest Academy of Economic Studies, Doctoral School, Romania, e-mail: catalina_oana_popescu@yahoo.com
} 
political interest (Bacharach and Baratz, 1970). These authors observe how the motivation of every form of action is a political one, to the point where certain aspects are deliberately kept off the decision agenda; those defining the decision agenda or the problem are therefore those who determine to a large extent the outcome of the decision. A more moderate view along the same lines states open problems, admitting that the main concern is a political one, in that information is still filtered and that political interests are the ones dictating negotiations (Pettigrew, 1973). All these aspects may be somewhat encompassed in a view of decision sequence: the fact that an authority makes a decision at one moment limits the decisions of an entire group at all subsequent moments, just as a decision an individual takes may limit all his/ her future options (an extreme example would be a decision to commit suicide successfully carried out which would end all future choices). We therefore consider appropriate to adopt a „bottom-up” approach - examine how individuals arrive at decisions and then how they adapt this process according to the others and their environment.

We will attempt to identify and briefly analyze the main barriers people may encounter in formulating and pursuing a well-structured decision-making model. This presentation is limited to individual decision-making and does not analyze decision-making in organization; such studies observe rather the interaction of individuals within an organizational environment with numerous and various stakeholders and a set of rules, regulations and guidelines. We structured the barriers for structured decision-making into factors affecting the objectives and criteria observed (unquantifiable factors), factors affecting the structuring of these objectives and criteria (oversimplified models that degenerate into paradoxes), and factors biasing the entire process and leading to unconscious ,,computing errors” (biases).

\section{Unquantifiable factors}

Loomes and Sugden proposed an entire theory of regret where the utility of the chosen alternative depended not only on the opportunity cost, but also on the utility of the alternatives sacrificed, through an effect of regretting a larger utility or, on the contrary, rejoicing in making a good choice (which would yield the greatest utility among available alternatives)( Loomes and Sugden, 1982). A function is then defined to give mathematical expression to this concept. Although the proposition seems intuitive and seems to convey a more accurate account of the utility actually felt by the decision-maker, transposing regret and joy into a mathematical function seems more of a scholarly exercise then a practical method. Furthermore, the perceived „success" of the choice influences the utility perceived after the actual decision has been taken - it would for instance be interesting to observe the measure in which decision-makers perceive a gain inferior to another potential gain as a loss. In estimating utilities modified by regret or rejoicing prior to the decision, anticipating either feeling would more likely act as a perceived grater stake in the decision and therefore create greater anxiety for the decision-maker. Moreover, if the consequences of an action are preferred to those of another action, this in itself would constitute the motivation of choosing one action over another, without a bonus or penalty of emotional affect. Regret or rejoicing would only motivate the decision-maker to make the best efforts in choosing wisely, that is to fundament his/ her decision thoroughly. Resented pressure, on the other hand, could lead to negative psychological states and therefore lead to a disutility of the decision process in itself.

Besides the relativity of the decision maker's position towards him-/ herself, there is the matter of the positioning with respect to other stakeholders. Wolff remarqued how "game theory is only capable of providing a formal analysis in situations where one pursues maximizing egotistical values" (Wolff, 1962). As a proposition to extend the model, he introduced the so-called contextual altruism as a case for dependency between the values obtained as a result of a game; in other words the utility of one player affects the utility of the other. This influence may be positive, if the player desires the other player's well-being, or negative if for whatever reasons the player desires the other player's downfall. In a simple case of altruism one player would replace his/ her own utility 
expected from a decision by that of the other player. Contextual altruism adds to the construct by introducing inter-personal compared utilities. This abstraction allows the consideration of costs associated with obtaining an expected result - if, for instance, by using the same means, the utility of one player is greatly superior to that of the other player, then he/ she would be less inclined to follow an altruistic principle, but would rather resent high personal stakes versus little relative impact on the other. Though this principle makes intuitive sense, giving it a numerical interpretation poses more problems. One option in this respect would be to introduce in the utility function of one player a special function $g$ such as

$$
U_{i}=f_{i}(\cdot)+g\left(f_{i}(\cdot), f_{j}(\cdot)\right)
$$

where $f_{i}$ and $f_{j}$ would be the egotistical utility functions of players $i$ and $j$ and $g$ is the impact of the relation between own egotistical gain and the gain of the other player.

In an extremely simplified but illustrative case, the function may be expressed as

$$
U_{i}=f_{i}(\cdot)+a * f_{j}(\cdot), a \in R
$$

Presuming that the players are benevolent towards each other and desire the good of one another. Then $a$ would be a positive parameter and $1 / a$ would express the level of damage that the player may accept to be inflicted onto the other player in order for him-/ herself to obtain one unit of egotistical utility. Similarly, if the players are malevolent towards each other (for instance rivals or otherwise enemies), then $a$ would be a negative parameter and $1 / a$ would represent the level of utility that one player may accept to be bestowed upon his/ her opponent in order for him-/ herself to obtain one unit of egotistical utility. The situation becomes more complicated to the point of paradox when the other player also implements a similar ,cross-utility” function. If the weight of this cross-utility is sufficiently high with respect to the egotistical utility within the overall utility (meaning if $a * f_{j}(\cdot) / U_{i}$ is significant), then we can observe an effect of circularity: one player knows that the other player wishes his benefit in a substantial degree, and that his benefit therefore greatly contributes to the other player's benefit. But the other player's benefit is also greatly important to the overall utility of the player in question. Therefore, if one player is greatly concerned with the other player's utility, and the other player is greatly concerned with the first player's utility, then for the sake of the other player the first player will pursue his own egotistical interest. This intuitive outcome may also be observed in terms of formal functions. May the following adjustments be made to equation (2) above:

We would then obtain

$$
\begin{aligned}
& U_{i}=f_{i}(\cdot)+a_{i} * U_{j}(\cdot) \\
& U_{j}=f_{j}(\cdot)+a_{j} * U_{i}(\cdot)
\end{aligned}
$$

$$
\begin{aligned}
& U_{i}=f_{i}(\cdot)+a_{i} *\left(f_{j}(\cdot)+a_{j} * U_{i}(\cdot)\right)=>U_{i}=\frac{f_{i}+a_{i} * f_{j}(\cdot)}{1-a_{i} * a_{j}} \\
& U_{j}=f_{j}(\cdot)+a_{j} *\left(f_{i}(\cdot)+a_{i} * U_{j}(\cdot)\right) \Rightarrow U_{j}=\frac{f_{j}+a_{j} * f_{i}(\cdot)}{1-a_{i} * a_{j}}
\end{aligned}
$$

We could make the further assumption that the players desire each other's benefit in a somewhat smaller degree then that of their own, meaning that in order to obtain one unit of utility they would accept that the other player would suffer disutility of several units, meaning $1 / a$, with $1 / a$ greater then 1 and therefore $a$ smaller then 1 . In this case

$$
a_{i}^{*} a_{j} \in(0,1)=>\left(1-a_{i}^{*} * a_{j}\right) \in(0,1)=>1 /\left(1-a_{i}^{*} a_{j}\right)>1 .
$$

Therefore, this mutual moderate consideration of the players for one another, expressed by positive and subunitary coefficients $a_{i}$ and $a_{j}$, would translate into a greater motivation for each 
player to enhance both him/ herself's, as well as the other's benefit. (as, as opposed to the former equation (2), the utility function is now multiplied by an supraunitary real number $1 /\left(1-a_{i} * a_{j}\right)$ ).

This model unfortunately proves unviable under the presumption that one player could only accept one unit of utility if this would not inflict upon the other player a disutility greater then $1 / a$ units, where $1 / a \in(0,1)$ and therefore $a>1$. In this scenario $\left(1-a_{i} * a_{j}\right)<0$, which would transform both utilities into disutilities, which does not make logical sense. Equations (5) and (6) would also be rendered unusable if $a_{i}=a_{j}=1$. In non-mathematical terms, this would mean that the utility of the other player is just as important to either player as his/ her own. Discussing the range of $a$, we would have to admit that a scenario where $a \in(0,1)$ would mean that the player would accept to „steel" utility from the other player at an inefficient „transfer rate" - he/ she would have the other suffer a disutility of several units so that he/ she could gain one unit of utility. Although plausible, such model contradicts in part the concept of altruism. We applied the limitation „,in part” as the very introduction of such a concept and such a coefficient as $a$ means that there are limitations to the length a player would go to satisfy his/ her own interest at the expense of others. This breach in morals cannot however be considered the norm, so we have to admit $a \geq 1$ as the base scenario, under which no mathematical conclusion can be reached. We should also mention that contextual altruism is a different construct from cooperation in the case of classical games. The latter express rather a joint effort in reaching egotistical ends, and competition is not excluded by motivations pertaining to the players, but it is rather avoided because the circumstances render it inefficient or ineffective. From an ideological perspective, the debate of contextual altruism is rich in arguments, from human capacity to fulfill oneself only inside society maintained by Aristotle to the consideration not of actions, but of their motivations (Schelling, 1968). However, as seen above, such a personal matter cannot easily be accommodated by mathematical models in a manner that is easy to understand and apply.

\section{Paradoxes - over-simplified models}

One of the more common criticisms to decision sciences is that „Knowledge on interaction between decisions, decision-makers and decision processes is extremely fragmented" (Davidsen, Spector şi Ullah, 2008). The implications of this accusation state that the models and methods developed so far do not address decision problems in an integrated manner from the multitude of perspectives that they face. In other words, the desire to provide a structured decision model despite the extensive description that characterizes each decision context led to unsatisfying simplifications. The faults of proposed models are apparent in the various paradoxes that they bear. Buridan's ass would die of hunger, incapable to choose between two alternatives of identical utility, be it two hay stacks of equal size situated at an equal distance from it. Confronted with choices between risk and uncertainty, some players would rather make suboptimal choices by any estimation rather than face uncertainty by making personal assumptions (Ellsberg, 1961). In another case known as the Newcomb paradox, two assessment methods lead to different conclusions (Nozick, 1969). In the Sankt Petersburg paradox, players would have to pay an infinity to compensate for the chances of winning. It would seem that playing with numbers give numbers free reign to play with us - ,If all we wanted was a way to pick one action from among many at any moment, then assigning real numbers (as opposed to some other ordered object) seems like overkill. And this is right - the great power of these results demands very strong conditions." (Easwaran, 2009).

The simple emergence of paradoxes is not in itself an indication of a deeply flawed or false theory. Logics, for instance, provides us with an entertaining list of such finds, starting from the undeterminable validity of the sentence „This sentence is false”. However, the range of paradoxes in the case of decision theory raises questions as to the pertinence of the models suggested, specifically expected value and expected utility.

The Sankt Petersburg paradox proposes a game where a coin is tossed; if the „tails" face appears, then the player wins 1 monetary unit, for instance one coin. If the ,heads" face appears, 
then the coin is tossed again. This time, if the ,tails" face appears, then the player wins 2 coins, and if the „heads" face appears, then the coin is tossed again, and so forth. Each time the ,heads" face appears, the prize doubles. The player receives the prize when the „tails" face appears and the game ends (Samuelson, 1960). If $1 / 2$ is the probability that either face appears on each toss of the coin, then the expected value $\mathrm{E}$ would be:

$$
\mathrm{E}=1 * \frac{1}{2}+2 * \frac{1}{2^{2}}+2^{2} \frac{1}{2^{3}}+\cdots=\frac{1}{2}+\frac{1}{2}+\frac{1}{2}+\cdots=\sum_{i=1}^{\infty} \frac{1}{2}=\infty
$$

The famous solution Bernoulli found was the introduction of the utility concept. This however addressed half of the problem, namely the part concerned with values, by attaching to it a more abstract construct, that of utility. This solution is not without downfalls, however, as the actual utility is filtered by human perception and therefore subjective and difficult to quantify. The other half of the problem is concerned with probability, rick and uncertainty. There are of course cases such as the tossing of a coin or of a dice where mathematical probabilities are obvious and there is only a matter of risk. Most often, however, decision-makers are faced with situations where uncertainty also lingers - that is, actual probabilities are unknown. This case, of both risk and uncertainty, is illustrated in the Ellsberg paradox.

A player would be presented with an urn containing 90 balls, out of which 30 red and 60 of other colors, either yellow or black. The player is asked to choose between two gambles: one where he/ she wins a prize if a red ball is extracted, and one where he/ she wins a prize if a black ball in extracted. The player is then asked between two other lotteries: one where he/ she wins a prize if either a red or yellow ball is extracted, and one where he/ she wins a prize if either a black or yellow ball is extracted (Ellsberg, 1961). Most participants to the experiment however chose the first gamble in the first case and the second gamble in the second case. In the first case, the player knew that there was exactly a $33.33 \%$ chance of a red ball being extracted, but did not know the chances of a black ball being extracted. Similarly, in the second case the player knew the chances of either a black or yellow ball being extracted were $66.66 \%$, but did not know the chances of either a red or yellow ball being extracted. So players most often fall back on those choices whose chances they can estimate. Instead, this proves a suboptimal choice under decision-theoretical analysis. Suppose that the player makes the presumption that there are more yellow balls then there are black, meaning there are more than 30 yellow balls and less than 30 black ones. Then in the first case, the player should chose the first lottery, and in the second case the first lottery. For an opposite assumption, the opposite applies. So under any assumption or distribution between black and yellow balls, adversity towards uncertainty prompts a suboptimal choice.

In dealing with both values/ utilities and probabilities/ risk/ uncertainty, mathematical decision models present therefore a weakness hard to overcome - that of subjective judgment that does not easily subject itself to mathematical formulation. In response, some models have been drafted based not on utility functions, but on preference and indifference relations between them to derive a total ordering (Easwaran, 2007). However, the conclusion reached until this point is that ,there probably is no hope of extending decision theory properly to a linear ordering on all actions" (Easwaran, 2007). The debate whether such an attempt should even be made knowing that in some cases decision-makers are genuinely indifferent between several alternative still stands. However, in arriving to such preferences a decision-maker still needs to construct criteria, estimate outcomes, and generally expose him- /herself to all sources of inadequacy that haunt expected utility models. An advantage of dominance-based theory remains that in replacing coefficients with preferences, it seems more intuitive and user-friendly.

\section{Biases as computing errors}

Cognitive biases represent thinking patterns based on observations and generalisations that can lead to memory errors, inexact judgments and flawed logic (Stanovich et al., 2008). Cognitive 
biases include, but are not limited to: hindsight bias (manifested through perceiving the inevitability of an event once it has occured), omission bias (tendency to omit information perceived as risky), confirmation bias (tendency to observe especially the information that confirms previously formulated conceptions and hypothesis) (Stanovich et al., 2008). In taking decisions, cognitive biases determine individuals to base their judgment excessively or mainly on previous expectations or knowledge and to not allow sufficient attention to information perceived as uncertain (Dietrich, 2010). Such individuals will not form a realistic overview and so the fundamentation of the decision is exposed to multiple risks of error. What is disconcerting is not that such phenomena occur, but that empirical studies have recorded that they occur on a large scale and even among the most educated (Yudkowsky, 2008), leaving some to wonder whether they represent the norm rather than the exception. Events such as economic bubbles and crashes and even political events are an additional argument in favor of the human mind's limitations and therefore its ability to make lucid, perfectly fundamented and objective decisions (Ex. Taleb, 2007).

\section{Empirical study}

In order to gather the opinions of real-life decision-makers on the obstacles they encounter, we deployed an online questionnaire asking respondents to simply list the main impediments or difficulties they face when taking decisions. For this purpose we employed the services of one of the free online survey platforms. In order to better analyze the phenomenon, the questionnaire also included demographic questions referring to age, level of income, tenure in the organization, educational level and marital status. Various business professionals from the consulting and banking industries in Romania, as well as researchers, were then invited via e-mail to complete the brief questionnaire, and as a result a number of 120 answers were collected for analysis. After reading all freely-formulated answers, we were able to identify three major areas of reference.

The first major concern respondents expressed was that of information availability or reliability. $32.5 \%$ or respondents specifically mentioned lack of information among the factors making decisions difficult. Another 5\% invoked correctness and precision of the information.

A second general issue that emerged was that of the complex relationship established with people around the decision-maker. This area was addressed by $30 \%$ of respondents. The exact manifestation of this issue, however, took very different forms, ranging from resentment of external intervention (in 15\% of cases) to conflict between own opinions and preferences and those of other people and social criticism (5\% of cases) and, on the other hand, concern for the decision's impact on others, especially dear ones, need or desire to consult with others and obtain consensus or approval (17.5\% of cases). This general factor can therefore have either positive connotations, when the decision-maker seeks support and concerns over the welfare of others, or negative connotations, if the decision-maker experiences or perceives social restraints.

A majority of $57.5 \%$ of respondents, however, still cited own inner conflicts as the main reason for which they feel some decisions as being difficult. $32.5 \%$ of respondents mentioned own indecision, hesitation and variability of preferences. Contrary to folklore, a majority of $56.4 \%$ of these were men, despite the fact that $54.17 \%$ of respondents were women. $10.85 \%$ mentioned the risks involved and own risk aversion as obstacles, while another $8.33 \%$ stated to fear the results of their own decisions. The conflict of emotional and rational aspects was also brought up by $7.5 \%$ of respondents. Interestingly, none of the respondents acknowledged manifesting cognitive biases or doubting the correctness with which they would define and implement a decision model; what all these claims refer to instead is the input data for such a model and the difficulty of accommodating sometimes conflicting desired outcomes.

Another $15 \%$ of respondents named time constraints as factors of complexity, mostly because such restrictions undermine a careful and thorough analysis of the situation and require intuition, experience or spontaneity that not all people are able to display all of the time. 
Although slight differences between demographic groups were inevitable, none of these were ample enough to be considered significant. One exception is the reaction to lack of accurate information $-51.11 \%$ of those complaining about such an obstacle were aged under 30 (in the total sample this demographic accounted for $47.5 \%$ of participants). A possible hypothesis to explain this phenomenon could be that decision-makers accumulate experience by repeatedly facing such a common shortcoming, experience materialized through both intuition and methods of minimizing the impact of the missing information in the decision model. In other words, in time decisionmakers acquire a certain immunity to this state of facts or at least become resigned to it.

To sum up, although objective factors such as pertaining to constraints of time and information are duly recognized as contributing to the difficulty of decisions, they are clearly outweighed by subjective factors characteristic of the decision-maker's own person and his/her relations to other people.

\section{Conclusions}

Albert Einstein has famously said that „Not everything that can be counted counts, and not everything that counts can be counted." This predicament is especially troublesome in the delicate art of decision-making, where the need to thoroughly fundament a choice in front of oneself and others is undermined by the relativity involved in this fundamentation. We have attempted to identify the main factors that may affect a decision model's adequacy in order to pave the way for further research on the influence each one has and on the way they interact. A short experiment has shown that decision-makers hardly complain about the difficulty of formulating a decision model, and only a minority of them finds it difficult to populate such a hypothetical model with necessary data within the given timeframe. Instead, the real challenge identified was of accommodating the diverging tendencies, preferences and conceptions governing themselves and their role in society in a manner that they may find hard to understand. Their quest is therefore not a mathematical or operational one, but rather of a psychological and even sociological nature, prompting for interdisciplinary studies. The findings of such thorough research could be used to redefine decisionmaking models in a manner that is both life-like and thorough and could therefore constitute a tool for both decision-makers and researchers in decision sciences.

\section{Acknowledgements}

This article is a result of the project POSDRU/6/1.5/S/11 „Doctoral Program and PhD Students in the education research and innovation triangle". This project is co funded by European Social Fund through The Sectorial Operational Programme for Human Resources Development 2007-2013, coordinated by The Bucharest Academy of Economic Studies.

\section{References}

1. Bachrach P, Baratz M.S., 1970. Power and poverty: Theory and practice. London, Unitek Kingdom, Oxford University Press.

2. Dietrich C., 2010. Decision Making: Factors that Influence Decision Making, Heuristics Used, and Decision Outcomes, retrieved from: http://www.studentpulse.com/a?id=180, lst consulted march 2011.

3. Easwaran K., 2006. Dominance-Based Decision Theory, retrieved from: http://www.ocf.berkeley.edu/ easwaran/papers/decisionslides.pdf, last consulted march 2011.

4. Ellsberg D., 1961. Risk, Ambiguity, and the Savage Axioms, Quarterly Journal of Economics, 75 (4), 643-669.

5. Loomes G., Sugden R., 1982. Regret Theory: An Alternative Theory of Rational Choice under Uncertainty, Economic Journal, 92 (368), 805-824. 
6. Nozick R., 1969. Newcomb's Problem and Two principles of Choice. In Rescher N. (ed.), Essays in Honor of Carl G. Hempel, Dordrecht, the Netherlands, Kluwer Academic Publishers.

7. Pettigrew A.M., 1973. The Politics of Organizational Decision-making. London, United Kingdom, Ed. Tavistock.

8. Samuelson P., 1960. The St. Petersburg Paradox as a Divergent Double Limit. International Economic Review, 1 (1), 31-37.

9. Schelling T., 1968. Game theory and the study of ethical systems, The Journal of Conflict Resolution, 12 (1), $34-44$.

10. Simon H., 1957. Models of Man. Hoboken, United States, John Wiley.

11. Stanovich K. E., West, R. F., 2008. On the failure of cognitive ability to predict myside and one-sided thinking biases. Thinking \& Reasoning, 14, 129-167.

12. Taleb N.N., 2007. Fooled by Randomness: The Hidden Role of Chance in Life and in the Markets. London, England, Penguin Books Ltd.

13. Wolff R.P., 1962. Reflections on game theory and the nature of values, Ethics, 72 (3), 171 179.

14. Yudkowsky E., 2008. Cognitive biases potentially affecting judgment of global risks, In Nick Bostrom N. and Cirkovic M.M. (eds.) Global Catastrophic Risk, Oxford, United Kingdom, Oxford University Press. 